
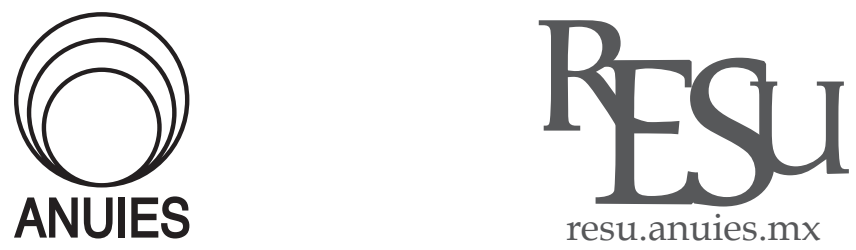

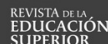

ARTíCULO

\title{
Expectativas de estudiantes universitarios indígenas en educación superior
}

\section{Pendiente}

\section{Gabriela Czarny*, Cecilia Navia** y Gisela Salinas***}

* Académica de la Universidad Pedagógica Nacional, Ajusco. Área Diversidad e Interculturalidad. Correo electrónico: gacza_2006@yahoo.com.mx

* Académica de la Universidad Pedagógica Nacional, Ajusco. Área Diversidad e Interculturalidad. Correo electrónico: ceeeci@yahoo.com

* Académica de la Universidad Pedagógica Nacional, Ajusco. Área Diversidad e Interculturalidad. Correo electrónico: gsalinas@upn.mx

Recibido el 27 de febrero de 2018; aceptado el 11 de octubre de 2018.

\section{Resumen}

Presentamos un avance del proyecto "Formadores de profesionales de y para la educación indígena. Retos para una descolonización académica", en la Licenciatura de Educación IndígenaUPN. Desde un enfoque cualitativo interpretativo y un cuestionario de preguntas abiertas, se analizan las expectativas formativas de los estudiantes y los saberes y prácticas de sus formadores, desde conceptos como descolonización de la educación superior, capacidad de agencia, formación centrada en los sujetos y reflexión entre pares. Los estudiantes esperan actuar de manera activa en su formación, desarrollar habilidades académicas y lograr el reconocimiento de sí mismos como sujetos educativos y de sus comunidades.

Palabras clave: Expectativas de Estudiantes Indígenas, Formadores de Profesionales Indígenas, Educación Superior, Prácticas de Formación, Descolonización. 


\begin{abstract}
We are presenting an advance of the Project "Trainer for professional to and for indigenous education. Challenge for academic des-colonization in the Indigenous Education Program at the UPN". From qualitative-interpretative perspective and an open questionnaire, formative expectations from students, knowledge and practices from their teachers are analized, from concepts like higher education des-colonization, agency, based on a subject-centered approaches and reflectiveness among peers. Students expect to perform in an active way in their training, develop academic abilities and achieve the effort to recognize themselves as educative subject and their communities.
\end{abstract}

Keywords: Indigenous Students Expectations, Trainer for Indigenous Professionals, Higher Education, Formative Practices, Descolonization.

\title{
Introducción
}

$\mathrm{E}^{\mathrm{s}}$ ste trabajo es parte del proyecto de investigación titulado: Formadores de profesionales de y para la educación indígena. Retos para una descolonización académica, que se desarrolla en la Universidad Pedagógica Nacional. Buscamos avanzar en la comprensión de algunos de los procesos formativos que se generan en la Licenciatura en Educación Indígena (LEI) de la Universidad Pedagógica Nacional, Unidad Ajusco, cuyos egresados se desempeñarán en el campo de la educación indígena escolar y comunitaria.

Nos centramos en el análisis de las expectativas formativas de los estudiantes de la LEI y en algunos procesos pedagógicos que establecen con sus profesores y con la institución. Procuramos avanzar en la identificación de saberes y prácticas de los formadores, que no suelen ser visibilizados desde el trabajo cotidiano de los docentes. Con ello intentamos aportar nuevas interpretaciones de las prácticas de formación que utilizamos en educación superior, en escenarios de diversidad sociocultural y lingüística, marcados por formas diversas de interpretar las historias de colonialidad. Partimos por considerar que sólo es posible desarrollar procesos educativos centrados en el estudiante, como sujeto portador de saberes y de expectativas propias, cuando el propio docente universitario ha pasado por un proceso formativo y reflexivo que le permite pensarse como sujeto en constante formación y aprendizaje.

En el marco del debate actual sobre el uso y la apropiación de los saberes escolares por parte de los pueblos indígenas, particularmente en relación con 
el acceso a la educación superior, se reconocen dos grandes tendencias: por un lado, las que resaltan que ese acceso por parte de jóvenes indígenas provoca el distanciamiento con la comunidad y la negación de su pertenencia a un pueblo originario; por el otro, algunos trabajos muestran que también han funcionado como espacio de "empoderamiento" para distintos miembros y proyectos de las comunidades. En este sentido, analistas de las políticas de educación superior para los pueblos indígenas señalan que "el acceso a la universidad por parte de los intelectuales indígenas actualiza una estrategia de apropiación y de empoderamiento ("empowerment") en un sistema de relaciones socioculturales altamente adverso para los pueblos indígenas" (Bello, en Rea, 2013: 45). Esto último se refiere a la crítica al conocimiento eurocéntrico, planteado como universal, desarrollado en las universidades donde frecuentemente se han negado los saberes y conocimientos de los pueblos; por ello, atender a los procesos formativos que desarrollamos en la educación superior con jóvenes indígenas, reconociendo distancias, cercanías y prácticas que nos convocan como sujetos reflexivos, es parte de nuestro objetivo en este escrito.

A continuación se presenta el marco donde se inscribe el debate de la profesionalización indígena en educación superior; posteriormente se expone el enfoque metodológico y analítico del trabajo, se realiza una descripción del tipo de programa que es la LEI de la UPN, y se presentan algunas de las perspectivas conceptuales que orientan el análisis sobre formación y lo que es enseñar y aprender en la educación superior. En la última parte del texto se expone el análisis de las expectativas de los estudiantes y luego las conclusiones.

\section{Educación Superior y Pueblos Indígenas}

En los últimos años el tema de la presencia, el acceso y la permanencia de jóvenes indígenas en la educación superior ha adquirido mayor visibilidad en la agenda educativa. En la región de Centro y Sur América se han impulsado diversos programas desde los que se han incorporado acciones afirmativas en universidades denominadas convencionales, así como la creación de programas específicos y de universidades denominadas interculturales dirigidas a población indígena. Sin embargo, contamos en México con diversos antecedentes en la creación de programas en educación superior dirigidos a la profesionalización de indígenas desde la década de los 70`s. En el diálogo construido entre académicos y activistas sociales indígenas y no indígenas desde ese periodo, la búsqueda por la reformulación de las condiciones de 
colonialidad que se consideraban vigentes en ese momento planteaban la imperante necesidad de que fueran los pueblos los que debieran definir sus políticas. En ese contexto, el escenario de la educación ya era visto como un posible conducto para la descolonización.

Es así que esos primeros programas, particularmente en el campo de la educación, la lingüística y la antropología, han marcado procesos para que los profesionales indígenas utilicen los conocimientos y las prácticas aprendidas en la academia a favor de los proyectos comunitarios y de las necesidades que plantean sus pueblos. De ahí que muchos egresados han contribuido a la conformación de liderazgos dentro de las organizaciones sociales indígenas; se han incorporado a la burocracia estatal o han continuado su formación buscando inserciones laborales diversas (Bartolomé, 2003; Pérez Ruiz, 2011).

Las demandas indígenas por mayor acceso a la educación superior se han convertido en un tema importante en el actual debate latinoamericano, pues representa para algunos una herramienta hacia la construcción de proyectos de autonomía y de mejora en las condiciones de vida individuales y colectivas. Aunque ha sido tradicionalmente un espacio de exclusión y de invisibilización de los saberes y prácticas de los pueblos indígenas, una serie de coyunturas locales, regionales, nacionales e internacionales vienen provocando un cambio en este ámbito.

La investigación en estas áreas ha avanzado en la documentación y el análisis de la implementación de algunos de los programas en el nivel superior que han aplicado políticas de acción afirmativa, tanto en las universidades convencionales como en las universidades interculturales (Mato, 2016; Mateos, Mendoza y Dietz, 2013). En los trabajos marcados por una definición intercultural en la educación superior se documentan algunos procesos caracterizados por una búsqueda de diálogo de saberes a partir de los inter-saberes y las prácticas inter-actorales, que se generan entre estudiantes indígenas con profesores no indígenas e indígenas y con referencia a la vinculación con los saberes y haceres comunitarios (Dietz y Mateos, 2010).

Otras investigaciones dan cuenta de los procesos referidos a las transformaciones de los proyectos individuales y colectivos de los jóvenes y las comunidades indígenas por el acceso a la educación superior, así como al sentido que se asigna a la profesionalización (Czarny, 2012; Rea, 2013; Santana, 2017). Mucho de lo que esos trabajos documentan, además de las tensiones que genera ser un/una joven universitario/a y adquirir nuevos hábitos vinculados a culturas juveniles, es el hecho de que en varios de los discursos de estos jóvenes aparece el sentido de estudiar para apoyar a sus pueblos y revertir injusticias y discriminaciones. No obstante, las áreas que requieren 
mayor indagación refieren tanto a las expectativas que tienen los jóvenes sobre las carreras que han cursado, el imaginario de futuro que se conforma en los espacios universitarios y sus posteriores inserciones laborales, así como el campo de la docencia universitaria y sus transformaciones en programas que trabajan con jóvenes indígenas.

Mato (2017) plantea que para la reformulación del trabajo en las IES que incorporan programas y población, en este caso indígena, más que buscar un "diálogo de saberes" se trata de avanzar en la construcción de modalidades duraderas y mutuamente provechosas de colaboración intercultural. Con esto refiere a que:

[...] no se trata simplemente de "dialogar" con el "otro", sino que se trata de hacer, de hacer juntos. Aún con todas las dificultades imaginables, dialogar no es el mayor reto. Es sólo un primer paso. De lo que se trata es de hacer juntos; y para esto hay que comenzar por reconocer que esto involucra personas e instituciones, no simplemente "saberes". Los "saberes" por sí mismos no dialogan (Mato, 2017: 15).

Con relación a esto último, partimos de la base de que se requieren dispositivos de formación que favorezcan nuevas formas de relación y de hacer juntos en las IES. Diversos autores han planteado como punto inicial, para un "diálogo intercultural", la incondicional reformulación de la situación de inequidad e injusticia (Tubino, 2007), y que no basta formular principios interculturales sino que se necesita una acción pedagógica reflexiva, crítica y comprometida. En este sentido, consideramos que se trata de lograr "traducir" y "accionar" permanentemente en los contextos de la interacción y de aprendizajes entre docentes y estudiantes, en este caso, juventudes indígenas provenientes de diversas comunidades y pueblos, hablantes algunos de sus lenguas originarias y otros no, así como con vínculos diversos con sus comunidades de procedencia.

\section{Enfoque metodológico y analítico de la investigación}

La perspectiva metodológica que orienta a esta investigación se ubica en el marco de los enfoques cualitativos de carácter interpretativo (Vasilachis, 2007; Mejía y Antonio, 2007), así como lo que se reconoce como diálogo colaborativo entre pares (Suárez, 2010), (en este trabajo los pares refieren a la relación entre profesores del programa). Nuestro punto de partida es lo que en las perspectivas de investigación colaborativa y narrativa se define como: “[...] espacios de trabajo colaborativo que se orientan a desarrollar y a poner 
a prueba formas de nombrar y considerar en términos pedagógicos 'lo que sucede' en los espacios escolares y 'lo que les sucede' a los actores educativos cuando los hacen y transitan (Suárez, 2010: 72).

Las diferentes perspectivas que se denominan interpretativa y narrativa - o biográfico narrativa - , son en gran parte herederas del giro hermenéutico y narrativo producido hacia finales de los años sesenta en el campo de la teoría de la investigación social (Castañeda y Navia, en prensa). Desde estos enfoques los fenómenos sociales y educativos no se entienden como "objetos" o "cosas" sino como "realizaciones prácticas" (Giddens, 1997) o como "textos a interpretar" (Ricoeur, 2001).

El análisis que se presenta tiene como una de las herramientas base cuestionarios de seguimiento y evaluación, aplicados a estudiantes de la LEI de diferentes generaciones, entre mayo y julio de 2017. Estos cuestionarios -34 en total - se repartieron entre los estudiantes de diversos semestres, aunque en su mayoría eran estudiantes del $8^{\circ}$ y alumnos que participaban en el programa de tutorías. Se mencionó que no era obligatorio que lo respondieran. El cuestionario contiene diversos tópicos elaborados para contar con evidencias escritas que den cuenta de algunas percepciones de los estudiantes sobre aspectos referidos a la licenciatura y a la UPN. En este trabajo se retoman principalmente los puntos referidos a las expectativas que tenían antes de entrar a la LEI, y cómo son éstas a la hora del egreso. La pregunta eje en este rubro fue: ¿Cuáles eran tus expectativas formativas al ingresar en la LEI-UPN y cómo son ahora?

El modelo que orientó el esquema para la sistematización de la información fue el de Meta perfil de educación para América Latina de Tuning (Montaño, 2013), y que a los efectos de esta investigación se reformuló en tres dimensiones: Profesional, Académica y Sociocultural. Si bien las dimensiones anteriores resultan campos interrelacionados, el objetivo de diferenciar aspectos permite reconocer demandas y expectativas específicas de los estudiantes.

Para el análisis de estos datos tomamos como referentes teóricos y conceptuales: a) las perspectivas sobre descolonización de la educación superior (Prada y López, 2009; Mato, 2016, 2017; Dietz y Mateos, 2010), b) las teorías que reconocen la capacidad de agencia (Arendt, 2001), c) la formación centrada en los sujetos (Honoré, 1992 y Navia, 2006) y en acompañamientos horizontales (Magnan, 1997), y d) las propuestas de análisis sobre docencia universitaria centradas en la auto-reflexión y la reflexión entre pares (Suárez, 2010). Asimismo, se recupera la reflexión - como ejercicio permanente- sobre la experiencia compartida como profesoras del Programa y como asesoras de los trabajos de titulación de los estudiantes de la LEI. Este último vínculo supone un contacto que suele generar un diálogo más intenso y directo con los asesoradas/os, en la construcción de sus objetos de indagación, 
mismos que conforman parte de sus intereses y procesos formativos, y en los que se ponen en juego procesos identitarios contrastantes, dilemas entre lo individual y lo colectivo, y entre diferentes enfoques de docencia y formas de interacción.

\section{Un programa universitario para formar a profesionales indígenas: LEI}

En 1978, cuando fue creada la Dirección General de Educación Indígena (DGEI) con el propósito de impulsar la educación bilingüe-bicultural, el subsistema de educación indígena experimentó un crecimiento sin precedentes al incorporar a jóvenes indígenas - que en su gran mayoría contaban con estudios de secundaria o bachillerato - para ser docentes en las escuelas primarias de ese subsistema. Con el apoyo de la Dirección General de Capacitación y Mejoramiento Profesional del Magisterio (DGCMPM), la DGEI impulsó la carrera de Profesor de Educación Primaria Bilingüe-Bicultural, conforme al Plan 1975 de educación normal básica, en el cual también se certificaban estudios de bachillerato; esta propuesta se ofreció en los estados con población indígena, a través de cursos intensivos a los docentes de educación indígena con estudios de secundaria.

En 1979, a partir de la preocupación para formar a profesionales que apoyaran el desarrollo de la educación indígena, el Centro de Estudios Superiores en Antropología Social (CIESAS), en vinculación con la DGEI y el Instituto Nacional Indigenista (INI), ofreció por dos generaciones la licenciatura en Etnolingüística. En 1980, el Centro de Integración Social (CIS), también en vinculación con la DGEI, coordinó la impartición de una generación de la Licenciatura en Ciencias Sociales, dirigida a profesores de educación indígena y, en 1982, la Unidad Ajusco de la Universidad Pedagógica Nacional (UPN), también en acuerdo con la DGEI, inició la operación de la Licenciatura en Educación Indígena (LEI) con el plan de estudios 1979.

Aunque nunca se firmó ningún convenio para poder operar la LEI, había un compromiso con responsabilidades compartidas: la UPN ofrecía una formación escolarizada y la DGEI otorgaba becas-comisión para que los directivos y docentes de educación indígena pudieran radicar en la Ciudad de México durante sus estudios. Al concluir la licenciatura y regresar a sus entidades, se esperaba que los egresados se incorporaran a las instancias técnico-pedagógicas, directivas y administrativas de sus estados para apoyar el desarrollo de la educación indígena. 
Los tres programas surgidos a finales de los años setenta eran una respuesta a las demandas de organizaciones indígenas en las cuales los profesores eran destacados participantes; de ellos, sólo la LEI continua vigente después de 35 años de operación y dos reestructuraciones curriculares: 1990 y 2011.

Además de la LEI, en 1990, en colaboración con la DGEI, la UPN empezó a ofrecer, en 34 Unidades de 16 entidades federativas, las Licenciaturas en Educación Preescolar y en Educación Primaria para el Medio Indígena (LEP y LEPMI), en una modalidad semiescolarizada, destinadas a la nivelación de profesores indígenas en servicio sin estudios de licenciatura (Salinas, 2011). A diferencia de las LEP y LEPMI, la LEI nunca se planteó nivelar a maestros en servicio; más bién se orientó a formar profesionales técnico-pedagógicos que, desde una perspectiva de los propios docentes indígenas y una preparación universitaria, pudieran contribuir a diseñar programas y, según la demanda planteada en esos momentos a la UPN por parte los maestros e intelectuales indígenas, estrategias descolonizadoras que les permitieran decidir la educación de los pueblos indígenas.

A la fecha la LEI ha formado a más de 35 generaciones de docentes, directivos y jóvenes indígenas. Muchos de los egresados se incorporaron como formadores en programas de formación y actualización de docentes indígenas; entre ellos destacan las LEP y LEPMI de la UPN y diversos programas educativos de Universidades Interculturales, creadas éstas por la CGEIB, a partir del 2004. También se han incorporado a Mesas Técnicas de la SEP tanto a nivel federal como estatal, a proyectos especiales para el uso de las lenguas indígenas en las escuelas, a los Centros de Maestros e instancias de dirección de la educación indígena a nivel regional, estatal e incluso federal. Una contribución que resulta importante destacar es su participación en la creación de las Normales Interculturales Bilingües en los estados de Oaxaca, Chiapas y San Luis Potosí, donde varios egresados de la LEI participan como docentes. También hay que señalar la inserción de nuestros egresados como docentes en escuelas de educación básica, sobre todo en indígenas y multigrado.

Para la UPN, ofrecer estudios de nivel superior a docentes y directivos de educación indígena implicó, desde las primeras generaciones, un fuerte desafío para los formadores para atender las necesidades de la educación indígena. A su vez, en la perspectiva de acercar la Universidad a la realidad educativa de los pueblos y comunidades indígenas, los tres planes de estudio que ha tenido la LEI han incorporado espacios orientados a la revisión y el reconocimiento de lo que ocurre en las comunidades, escuelas y aulas de educación indígena, destacando entre ellos el desarrollo de los estudiantes de prácticas o trabajo de campo. Esto ha exigido a un grupo importante de 
los académicos que se han involucrado a lo largo de los años en este Programa, el desarrollo de investigaciones en escuelas y comunidades indígenas y la participación en diferentes procesos de formación y actualización de los docentes, directivos y formadores de docentes indígenas en servicio.

Como hemos señalado, la LeI está vinculada a los reclamos de diferentes organizaciones indígenas que señalan que sus profesionales y docentes deben contar con una formación específica, que reconozca sus derechos y que posibilite orientar y mejorar la educación de niñas, niños, jóvenes y adultos indígenas (Rebolledo, 2014). Las prácticas de formación heterogéneas, que desde su creación se han ido desarrollando por los maestros y alumnos, nos permiten hablar de que se producen en cierta manera formas de "indianización" de las aulas de la upN Ajusco, en el sentido de que ha permitido la visibilización, el reconocimiento y el auto-reconocimiento de jóvenes docentes y bachilleres originarios de diferentes pueblos y comunidades indígenas. Pero así como se ha posibilitado el intercambio entre estudiantes con distintas lenguas y culturas que han enriquecido la vida universitaria, al mismo tiempo parece que aún prevalecen sedimentadas formas de relación de los formadores con los estudiantes indígenas de tipo directivo y heteroformativas. Existen algunas prácticas como discriminación y paternalismo, presentes no sólo entre profesores y estudiantes sino también entre los mismos estudiantes universitarios.

Los cambios experimentados en el curriculum han implicado fuertes discusiones y trabajo colaborativo entre el equipo docente, vinculados a las perspectivas de la educación indígena y a los enfoques intercultural y bilingüe. La última reestructuración curricular (2011) contó incluso con una amplia participación de los estudiantes, quienes buscaban contribuir a que sus preocupaciones fueran consideradas como una cuestión de derecho. Los campos de formación profesional propuestos en el Plan 2011 también se vincularon con retos que enfrenta la educación indígena: la educación bilingüe, la educación en las aulas y la formación de docentes para la educación indígena, y la educación comunitaria en contextos multiculturales.

A partir de 1996, el otorgamiento de becas-comisión por parte de la DGEI empezó a obstaculizarse, afectando la posibilidad de que maestros estudiantes de la licenciatura pudieran continuar sus estudios. Sin embargo, algunos estados como Oaxaca, Chiapas, Guerrero y Chihuahua permanecieron apoyando a los maestros hasta hace algunos años. La reforma educativa del 2013 terminó con estos apoyos y sólo unos cuantos docentes del estado de Oaxaca, que están por concluir sus estudios, tienen algún respaldo en acuerdo con sus autoridades. Esta situación, después de varias décadas de presencia de 
docentes indígenas en las aulas de la Unidad Ajusco de la UPN, ha implicado un fuerte cambio en el perfil de los estudiantes.

\section{Retos para las universidades y la docencia del siglo XXI}

Si bien en el contexto actual se plantea que la docencia universitaria integre el desarrollo de competencias para la "apreciación y el respeto de la diversidad y la multiculturalidad" (Montaño, 2013), creemos que las universidades se encuentran lejos de lograrlo, pues esto exigiría desplazamientos de una educación centrada en una enseñanza con una fuerte visión monocultural, eurocentrada, hacia una con una visión inter-intracultural con sentido crítico, que cuestione los modos en que se ha producido el conocimiento y lo que ello ha generado en tanto legitimidad para la exclusión de otros saberes y prácticas (Prada y López, 2009). Esto implicaría no solamente asumir nuevas prácticas de enseñanza, sino también de relación con los estudiantes, que partan del reconocimiento del otro como sujeto educativo, portador de saberes, de formas de aprender y de producir el conocimiento.

Nos referimos entonces a que la universidad se enfrenta también a la necesidad de aperturar su relación con el/los saber(es) y con la/s epistemología(s) en una perspectiva plural. Lo anterior, en cierta manera, confronta a los principios con los que fueron fundadas las universidades (López y Prada, 2009) y a la misma noción de conocimiento científico que viene casi instalada como "una verdad", pero que comienza a ser cuestionada en el sentido de problematizar lo que es la ciencia y lo que es producir conocimiento en una visión más amplia en contextos de transformación de los ciclos con el saber, mediados por diversas tecnologías.

En los últimos años es común encontrar, en el campo educativo y en las perspectivas sobre enseñanza y aprendizaje, un discurso que promueve la autonomía, la formación centrada en los sujetos, la autoformación (Navia 2006; Montaño 2013). Si bien la enunciación de estas categorías no significan cambios inmediatos o sustanciales en las prácticas de formación de los estudiantes, podemos señalar que lentamente van ocupando el mundo de creencias que marcan la vida cotidiana de las instituciones, así como van generando nuevas prácticas formativas y nuevas formas identitarias de los docentes.

En las perspectivas mencionadas se aboga por el pasar de figuras tradicionales en las que el/la docente era o es el que enseña, a facilitador de los aprendizajes de los estudiantes. Lo anterior implica otorgar un peso importante de la formación a estos últimos, como actores y autores de su formación. Este proceso no ocurre sin tensiones, pues implica actuar en sentido 
contrario a prácticas sedimentadas tales como establecer una relación directiva con los alumnos, que caracterizan las formaciones altamente estructuradas que permean nuestras instituciones.

Que el estudiante se transforme en (ser) actor de su formación implica, entre varios aspectos, asumirse como autor de su propia formación, así como dar cabida a nuevas formas de construcción de sí mismos y de interactuar con los otros. Esto es posible si se parte del reconocimiento de sí, como sujeto educativo y como sujeto proyecto, que actúa desde y hacia la autonomía y la libertad. Reformular la noción de sujeto educativo y postular el horizonte de sujeto proyecto, implica nuevas prácticas de formación, de los formadores y los estudiantes, "en las que se invita a cada uno a una reflexión sobre su vida, poniendo en cuestión la autoridad del saber y la autoridad del formador, y correlativamente transformando su medio ambiente educativo, su espacio personal y socializado" (Honoré, 1992: 28).

Las trayectorias formativas de una parte importante de los estudiantes de la Licenciatura en Educación Indígena nos muestran que han recibido una atención educativa heteroformativa, caracterizada en una alta proporción por experiencias de discriminación y exclusión educativa. Algunos trabajos sobre trayectorias escolares de estudiantes indígenas en la LEI y en las LEP y LEPMI muestran que las escolaridades previas están marcadas no sólo por tiempos interrumpidos en los estudios, sino por una diversidad de instituciones escolares que se crearon en el medio indígena para "compensar" la ausencia de propuestas educativas, pero que no siempre lograron consolidarse, como por ejemplo, telesecundarias, telebachilleratos, etc. (Salinas, 2011; Czarny, 2012; Coronado, 2016). Al mismo tiempo, esos trabajos muestran las profundas desigualdades e inequidades en las que transcurrieron los procesos vitales y formativos de los estudiantes.

En diversos relatos de estudiantes indígenas se identifican recuerdos positivos y valiosos de sus procesos escolares previos a la universidad, pero también encontramos imágenes cargadas de profundas relaciones de discriminación y racismo en contextos rurales y urbanos, y relaciones jerárquicas y verticales en la escolarización, particularmente en la relación maestro-alumno (Czarny, 2012). Este último aspecto tiene diversas implicaciones - junto a otros elementos - como la imagen en la que el docente es el que "debe mandar e impartir la clase", y el estudiante el que debe responder a esos requerimientos.

La ubicación de que el docente es el que "sabe" y el que pone las reglas para habilitar o deshabilitar discursos, saberes y prácticas legítimas en los procesos escolares y de aprendizaje, traspasa al imaginario y a los escenarios de cómo debe enseñarse y aprender en la educación superior, aspectos 
que en algunos casos se logra desestructurar en el ámbito universitario en las relaciones maestro-alumno. Sin embargo, la idea de que el maestro es o debe ser el centro de la enseñanza y por eso "manda", sigue de algún modo presente, al mismo tiempo que genera procesos autonómicos para el aprendizaje, lo cual representa frecuentemente una tensión en los procesos de enseñanza y de acompañamiento.

Para comprender la configuración de nuevas formas de relación con nuestros estudiantes de la licenciatura de educación indígena, nos resulta útil la noción de acompañamiento, que supone permitir al estudiante, en su condición de aprendiente, a que se exprese, recupere su experiencia y utilice lo que ha comprendido para dar sentido a sus aprendizajes (Magnan, 1997). El acompañamiento es un dispositivo para la formación de apoyo, colaboración y ayuda centrado en el estudiante. Supone una relación de cercanía que vaya en la misma dirección del estudiante, y una relación de horizontalidad, de intercambio de saberes, de apertura a espacios de reflexión, que reconozca que en este proceso formativo existen tensiones y conflictos con el conocimiento y con los sujetos.

Como formadores, transitar de prácticas de formación altamente estructuradas a otras centradas en el acompañamiento y la autoformación implica abandonar prácticas verticales (que van desde las exigencias que van más allá de las posibilidades de los estudiantes a las exigencias que minimizan sus posibilidades formativas). Lo anterior se refiere, por ejemplo, a no considerar las trayectorias de inequidad previas a la universidad, o por el contrario considerarlas de tal forma que se disminuyen las demandas y se relajen las exigencias académicas.

Partimos de considerar que sólo es posible desarrollar procesos educativos centrados en el estudiante, como sujeto portador de saberes y de expectativas propias, cuando el propio docente universitario ha pasado por un proceso formativo y reflexivo que le permite pensarse como sujeto en constante formación y aprendizaje.

\section{Expectativas de los estudiantes indígenas sobre la profesionalización}

El análisis de las respuestas que los estudiantes dieron en torno a las expectativas de sus estudios en la Licenciatura en Educación Indígena nos permitieron formular trece categorías. Para agruparla en una dimensión más amplia recuperamos las dimensiones Profesional, Académica y Socio-cultural pro- 
puestas en el Modelo de Meta perfil de educación para América Latina de Tuning (Montaño, 2013).

La construcción de las 13 categorías que corresponden al tipo de expectativas de los estudiantes de la licenciatura se realizó haciendo uso del análisis de contenido de las respuestas. (ver Tabla 1). Cabe aclarar que como toda clasificación, varias categorías se encuentran interrelacionadas, pero a los efectos del análisis las agrupamos de este modo para clarificar de la mejor manera la intención de las respuestas proporcionadas.

Tabla 1

Dimensiones y categorías de las expectativas formativas en la LEI

\begin{tabular}{lll}
\hline \multicolumn{1}{c}{ Dimensión Profesional } & Dimensión Académica & Dimensión Sociocultural \\
\hline $\begin{array}{l}\text { - Se amplía la visión de la } \\
\text { profesión }\end{array}$ & - Conocimiento & - Procesos identitarios colectivos \\
- Ejercer la docencia & - Capacidades críticas & $\begin{array}{c}\text { Ayudar y aportar a la } \\
\text { comunidad }\end{array}$ \\
- Ser un profesional & - Identidad como estudiante & - Descolonización \\
- Pedagógico-didáctica & universitario & \\
- Revaloración del trabajo de & & \\
la educación & & \\
\hline
\end{tabular}

\section{Dimensión profesional}

En las respuestas de los estudiantes de la LEI se expresa un interés por estudiar con fines profesionales, aunque la idea de la profesión parece tener diferentes orientaciones así como reconfigurarse a lo largo de sus estudios. En parte, este proceso ocurre no sólo de manera individual sino también de forma colectiva. La LEI se les presenta a muchos como un espacio que les permite reconocerse de un modo distinto al que han tenido en experiencias previas a su inserción a la universidad. Así, algunos estudiantes afirman que la licenciatura es como "una familia en la cual están contigo, te apoyan, te escuchan". El ser reconocidos como sujetos académicos por sus docentes les permite reconocerse a sí mismos como sujetos educativos y con derecho a ocupar el espacio institucional que los recibe, en este caso la UPN.

Encontramos que varios estudiantes ingresaron a la LEI con el interés de ejercer la profesión docente. Algunos señalaron la docencia de manera general, aunque otros especificaron la educación bilingüe, la educación con sus culturas y trabajar como docentes en sus comunidades de origen.

Al referirse al ejercicio de la docencia, se señaló la necesidad de crear sus propios recursos educativos, elaborar materiales retomando sus necesidades y el contexto, sus costumbres, tradiciones y la lengua, y tener las he- 
rramientas necesarias para desarrollar un curriculum pertinente: "encaminar a la educación con propuestas pensando en la comunidad [desde nosotros]".

Si bien una parte de los estudiantes manifestaron tener interés por estudiar la licenciatura con la intención de trabajar como docentes, encontramos que en el trayecto de su formación estas expectativas fueron cambiando y ampliándose. Varios estudiantes señalaron que la idea de formarse como docentes fue perdiendo fuerza en la medida en que avanzaban sus estudios y en la que fueron profundizando sus conocimientos sobre el campo de la educación indígena. En una de las respuestas se señaló: “[...] que la cultura no se remite tan sólo a la educación bilingüe, que la educación indígena puede desarrollarse en varios ámbitos y que es posible también aportar a otros campos". Otras respuestas mostraron el cambio de una posición negativa respecto a la docencia a una imagen positiva, lo que nos permite suponer que algunos estudiantes ingresaron a la licenciatura sin tener interés por la docencia, pero que en la medida en que se fueron acercando al tema docente, se fueron sensibilizando y configurando expectativas favorables en torno a este tipo de ejercicio profesional, sea en la educación en general o en la educación indígena.

Lo que encontramos es que, si bien la LEI no se formula como un espacio de formación docente, los estudiantes manifiestan una tensión entre sus expectativas de ejercicio profesional entre la docencia y aquéllas ligadas a las diferentes opciones de trabajo que la licenciatura les ofrece, que contrastan con las posibilidades de inserción laboral en diversos ámbitos educativos, a nivel nacional y de sus comunidades.

\section{Dimensión académica}

Como hemos señalado más arriba, la LEI se configura con un espacio en el que a los estudiantes les es posible reconocerse como sujetos educativos de otro modo, es decir, al margen de las historias de exclusión y discriminación que una gran parte de ellos han vivido, tanto en las instituciones educativas como fuera de ellas, antes de entrar a la universidad. De ahí que en las respuestas de los estudiantes se presenta el interés de asumirse como sujetos académicos, lo que expresan como el deseo de ampliar sus conocimientos, desarrollar habilidades en la escritura académica, desarrollar capacidades críticas e identificarse como estudiantes universitarios.

Al igual que se ha encontrado en diversas investigaciones de educación superior (Navia y Hirsch, 2015), para los estudiantes de la LEI obtener nuevos conocimientos se ubica como la prioridad más importante. Destaca en las 
respuestas una fuerte orientación a conocer temas vinculados a la educación comunitaria, lo que se expresa en respuestas como: "conocer a los pueblos originarios", "conocer la historia de los pueblos originarios", "conocer las culturas", "conocer más de las comunidades", "ser formados en la cultura", “conocer las lenguas y la organización entre sí". Este elevado interés por sus culturas y pueblos puede ser un indicador que nos muestre el efecto que tiene el curriculum en la configuración, el fortalecimiento y el reconocimiento de sus pueblos y su pertenencia a los mismos. En las respuestas los estudiantes mencionan en menor medida los conocimientos ligados al proceso de enseñanza y aprendizaje.

Otra cuestión que concentra las expectativas de los estudiantes en la licenciatura está referido a la constitución de su identidad como estudiante universitario. Nos referimos con ello al hecho de que varias respuestas giran en torno a "ser estudiantes universitarios", "fortalecerse como estudiantes", "destacar en sus estudios", "valorar su formación" y "continuar sus estudios". En diferentes oportunidades de trabajo en el aula hemos podido observar que nuestros alumnos se reconocen en esta licenciatura por primera vez como sujetos activos de su propia formación. De ahí que ser "estudiante universitario" constituye de por sí no sólo un logro significativo en su trayectoria formativa, sino también un punto de inflexión en su constitución como sujetos capaces de ejercer el derecho de ocupar un espacio en la educación superior. Esto se manifiesta en el hecho de lograr desplazarse de un espacio de no-reconocimiento o de invisibilización de sus posibilidades educativas en espacios formativos previos (en educación básica y educación media superior), a uno en el que puedan realizarse, constituirse en una dimensión autoformativa, pero también inter-formativa, en la relación con otros, sean éstos otros estudiantes indígenas, no indígenas o con profesores.

Entre otros aspectos, encontramos que el tema de la alfabetización académica también ocupa un lugar importante como expectativas de los estudiantes, en particular a nivel de expresión y comprensión de los textos académicos, sobre todo en la escritura, la comprensión lectora y la expresión oral. Al respecto vale mencionar que es frecuente encontrar en algunos estudiantes importantes problemas en la escritura y la lectura académicas, que se presentan como obstáculos importantes en su proceso formativo. Por ejemplo, en una sesión de tutoría, un estudiante refirió "quiero que me ayude a escribir para poderme sentir como estudiante de la universidad, para no tener miedo a presentar mis trabajos escritos a los maestros" (estudiante de cuarto semestre).

Otra expectativa de los estudiantes es la de desarrollar capacidades críticas, reflexivas y participativas. Podemos señalar que la preocupación por desarrollar capacidades críticas está vinculada al problema de la escritura 
pero también a su identidad como estudiantes. El interés por desarrollar capacidades críticas y reflexivas nos remite por un lado a expectativas externas, como por ejemplo las demandas de tareas de sus profesores que les exigen cada vez más trabajos escritos o intervenciones con mayor nivel de profundidad o complejidad. Sin embargo, también podemos distinguir que en esta expectativa está presente una aspiración por mejorar su condición como estudiantes, en tanto sujetos académicos y posicionarse como tales tanto en el espacio universitario como durante su estancia en el mismo y fuera de éste una vez que egresen.

No es menos importante que en las respuestas de los estudiantes se expresen estas necesidades, pues de ser atendidas, pueden tener efectos formativos importantes en su egreso como profesionales.

\section{Dimensión sociocultural}

En esta dimensión, encontramos cuatro elementos que impulsan a los estudiantes a ocupar de otro modo el espacio universitario: procesos identitarios y colectivos, la relación con la comunidad, procesos de descolonización y procesos de configuración de la identidad personal.

El más importante, y segundo en importancia en las expectativas generales de los estudiantes (el primero se refería a ampliar sus conocimientos), tiene que ver con el desarrollo de procesos de fortalecimiento y/o reconocimiento de las adscripciones comunitarias y colectivas. Destaca en particular una afirmación de un estudiante sobre su trayecto formativo en la universidad que sintetiza gran parte de las respuestas referidas a este tema: Aprendí a formar mi propia identidad, con mi misma comunidad, darme cuenta y valorizar los saberes que aún seguimos conservando. Me gustaría hacer algo para que no se pierdan.

En esta respuesta, se expresa una forma de resignificar el sentido de su formación ligado a la revalorización y resignificación de sí mismos, como sujetos ético-políticos. Es decir, además del reconocimiento de sí mismos, en su vinculación con sus comunidades, y por ende con sus culturas y saberes, está presente una re-apropiación de la capacidad de actuar en su realidad. De ahí que encontramos, junto con esta respuesta, otras que expresan: "revalorar los conocimientos", "fortalecer los procesos culturales, lingüísticos e identitarios", "concientización o valorización de aspectos culturales".

Otro estudiante nos aporta de forma más profunda el sentido que le otorgan a lo que mencionan como "valorar" o "valorizar" en las expectativas de los estudiantes: Valorizar, respetar, conocer y comprender las formas de vivir, de 
ser, de aprender, de pensar, de actuar de las comunidades indígenas, de los miembros de las comunidades. Encontramos en este texto un profundo impacto formativo de sus estudios, pues no sólo incluye la referencia a su cultura, sino la que les toca a sí mismos como actores de su formación, en una dimensión de autoreconocimiento y autoafirmación personal.

La respuesta también nos remite a un reconocimiento de sí mismos como agentes educativos y como sujetos colectivos. Vivir, ser y pensar desde sí mismos adquiere una connotación de estar presentes en el mundo, como sujetos históricos, de manera activa. De ahí que se puede señalar que en la LEI, algunos estudiantes encuentran un espacio que les da posibilidades como agentes educativos con una dimensión ético-política respecto a su profesión en el campo de la educación indígena.

Ligado a este aspecto, encontramos respuestas referidas a un principio profesional de beneficiar a las comunidades, en particular las suyas, así como también la idea de un retorno a las mismas. La idea de retorno, en este caso, remite a "regresar" a trabajar a las comunidades de procedencia. Sin embargo, y por diversas razones, también es un elemento que se cuestiona entre los estudiantes y se reconforma en ideas como "trabajar para los pueblos", más allá de regresar o no a las comunidades de origen Si bien no podemos señalar que esto ocurre en todos los estudiantes, las siguientes expresiones son representativas de este sentido de compromiso ético hacia sus comunidades: "ayudar a las comunidades originarias en el ámbito educativo", "hacer proyectos que pueden reflejarse en la comunidad", "trabajar en proyectos alternos en beneficio de las comunidades indígenas", "reflexionar y proponer alternativas de la situación educativa de nuestro pueblo y país", "participar en proyectos comunitarios en los pueblos indígenas" y "volver a la comunidad para trabajar con ellos." Esto indica que los estudiantes esperan que su carrera les ofrezca una profesión que pueda responder a las necesidades educativas de sus contextos de origen $u$ otros contextos.

La universidad y la LEI permiten a muchos estudiantes resignificar el lazo con sus comunidades y la pertenencia a sus culturas. En cierta manera, el espacio formativo se convierte en un espacio de retorno hacia sí mismos, como sujetos individuales y como sujetos colectivos. Se da lugar con ello a ciertos desplazamientos de significados, que les permiten romper con formas incorporadas de desigualdad, de negación de su capacidad de autonomía y de autoreconocimiento, de discriminación, normalizadas a lo largo de su trayecto formativo previo. Nos referimos tanto a los trayectos de escolarización, desde educación básica a educación media superior, así como a los trayectos educativos en sus contextos, en donde estas formas de desigualdad también han estado presentes. Por ejemplo, algunos estudiantes han señalado que no son 
hablantes de la lengua de sus comunidades porque sus padres no quisieron enseñarles (la lengua). El argumento más frecuente que se menciona es que hablar la lengua indígena los coloca en situación de discriminación. Con ese mismo argumento, también se ha señalado que en diversas comunidades algunos padres de familia exigen que en las escuelas no se use la lengua indígena.

Como hemos señalado al describir el inicio de la LEI en la UPN, las expectativas planteadas en ese momento, por parte de los representantes de las comunidades indígenas, fueron que se generaran profesionales de la educación comprometidos con un proyecto de descolonización. Distinguimos, en el discurso de dos estudiantes, algunas manifestaciones de estas expectativas: "La historia de nuestros pueblos originarios oculta y renegada, aquí da vida y se hace presente en cada uno de nosotros" (Estudiante $8^{\circ}$ semestre). "Ahora pienso que no hay nadie mejor que conozca sus comunidades como nosotros mismos y qué cosas podemos llevar a cabo para defendernos" (Estudiante de $8^{\circ}$ semestre).

Creemos que detrás de estas expresiones están presentes proyectos de los estudiantes, como agentes educativos de sus comunidades, marcados por formas de autodeterminación y actualización de su realidad a los tiempos actuales. Se trata de encontrar en la licenciatura un sentido que pueda conducir a asumir "una historicidad propia, una descolonización de los imaginarios y de las formas de representación" (Rivera, 2010: 53), que han caracterizado sus trayectorias educativas y escolares. En ese sentido, el proceso de formación les ha permito descubrir su "posibilidad de elección y de proyecto" (Honoré, 1992: 29). Esa elección de ser sujeto proyecto, en la licenciatura adquiere entonces dos connotaciones: un sentido de lo colectivo, y otro, aunque muy poco mencionado por los estudiantes, pero que consideramos condición de la primera, un sentido personal: "prepararme para ayudarme a mí misma" y "me ayudó, en primera instancia a encontrarme a mí".

\section{Conclusiones}

Reorientar las prácticas formativas, recuperando la reflexión de los estudiantes sobre sus experiencias escolares, y la intervención, mayor o menor, o negada en sus procesos académicos previos, constituye una de las acciones que comienzan a develarse en el Programa.

El proceso reflexivo implica abandonar la idea que considera que la formación es lo que nosotros como docentes hacemos, sin un proceso por el cual los estudiantes se comprometen en ella. Las experiencias educativas previas de nuestros estudiantes revelan la presencia de huellas formativas profunda- 
mente ligadas a la negación y el silenciamiento de sus culturas y lenguas, y de sí mismos, en su condición de actores históricos y educativos.

Hablar de procesos formativos y expectativas de estudiantes en un programa como la LEI implica reconocer los procesos históricos que han venido impactando - con diferentes actores indígenas y no indígenas - en la profesionalización indígena, en este caso en el campo educativo. Con lo anterior, no negamos que existe a nivel de la educación superior lo que algunos autores señalan como violencia simbólica en las instituciones frente a las prácticas y epistemes de los pueblos indígenas (Zapata, 2017). Sin embargo, reconocer la historia y las luchas para construir y sostener espacios para los pueblos indígenas, en este caso como la LEI, forma parte de los aprendizajes, diálogos y silencios que marcan a las instituciones y a todos sus actores.

Podría argumentarse que la LEI es un programa de educación superior vinculado en sus orígenes al impulso del Estado de "dar respuesta" a las demandas de los pueblos, en este caso, para el acceso y la formación de profesionales en el campo de la educación indígena. Sin embargo, como podemos apreciar en estre trabajo, es un programa en el que las comunidades han puesto interés y han incidido en cierta manera en su orientación, lo que ha permitido a sus egresados irse apropiando, de distintos modos, del discurso sobre este campo educativo. Con el tiempo, pueden reconocerse algunos rasgos que señalan transformaciones académicas en el Programa, tema que intentamos ubicar en las prácticas de los formadores de profesionales indígenas, a través de algunas de las expectativas de estudiantes indígenas.

Claudia Zapata (2017) señala que, como parte de las luchas anticoloniales que los intelectuales indígenas en las Américas (ubicados así por su condición de profesionales y universitarios) han realizado, se encuentran aquellas que ella denomina "procesos de subversión". Éstas se han desarrollado a través de prácticas aprendidas en la academia, como la apropiación, la construcción y la utilización del discurso y las herramientas académicas para luchar contra la hegemonía del discurso del mestizaje; o lo que otros indican como "utilizar las mismas herramientas impuestas por el colonizador ahora para liberarnos".

En relación con dichas perspectivas, pueden identificarse, en las expectativas de los estudiantes de la LEI, algunos rasgos que dan cuenta de múltiples procesos vinculados con la transformación individual y colectiva que experimentan en su formación. En este sentido, como se ha mostrado, existen algunas experiencias y prácticas en la LEI que favorecen a la restitución de las visiones negativas y negadas que los estudiantes han construido en experiencias formativas previas. Como sujetos sensibles y afectivos, el miedo y la inseguridad se han presentado en cierta manera como una barrera para 
la posibilidad de autorizarse como actores y autores de su propia formación. Esto constituye posiblemente una de las principales apuestas como formadores, en tanto se trata de mirar o recuperar, entre nuestras prácticas, aquellas que movilizan a los estudiantes para reflexionar sobre experiencias, creencias y supuestos que les niegan su capacidad de actuar y han interiorizado en sus trayectorias escolares previos.

Sin embargo, en su trayecto universitario, desde sus palabras, hemos logrado apreciar que, en cierta medida, sus estudios los ponen en una condición de sujetos activos con diversas perspectivas y posibilidades en sus proyectos personales y comunitarios. La experiencia en el programa les permite recuperar historias sociales y culturales que fueron minorizadas y negadas en las presentes "estructuras neocoloniales que aún mantienen los Estados nacionales" (Bartolomé, 2003), aspecto que potencia sentidos individuales y colectivos, y la generación de propuestas conforme a las necesidades y demandas de los pueblos indígenas para el campo educativo.

La misma situación referida al tipo de programa que es la LEI en la UPN, Ajusco - formar profesionales para la educación indígena-, genera algunas tensiones en los imaginarios de los estudiantes, quienes en muchos casos ingresan con la idea que se formarán para ser profesores, y al mismo tiempo permite recuperar a otros nuevas perspectivas sobre qué es ser docente y un profesional del campo educativo que responda a las demandas de los pueblos indígenas en los actuales contextos.

Es importante reconocer que existen fuertes expectativas entre los estudiantes por adquirir conocimientos y habilidades como cualquier estudiante universitario que busca un título para afrontar su futura condición de profesional. En este sentido, las dimensiones académicas que esperan obtener en su trayecto de formación en la LEI, como capacidades analíticas, críticas y de desarrollo de la escritura, resultan retos fundamentales. El tema de la escritura académica en programas donde una parte de su estudiantado es hablante de una lengua originaria - como lengua materna - , y quienes han tenido procesos desiguales y de fuertes inequidades en sus escolarizaciones previas, representa importantes retos para el trabajo académico en la educación superior. Si bien este tema no aparece como referido específicamente en las expectativas, se reconoce entre los docentes y los estudiantes que aún hay mucho por hacer en este rubro, no sólo en programas como éste, sino en toda la educación superior a la que asisten jóvenes indígenas. Estamos en la lógica de que la transformación nos implica a todas y todos, y es una lucha que requiere de varios frentes y redes de colaboración, así como de diversos procesos de revisión y actualización reflexiva de nuestras prácticas académicas y, en eso, creemos, estamos. 
Se plantean entonces para los formadores tareas complejas como repensar las universidades y su relación con ellas, el papel de los enseñantes y la relación con sus estudiantes, lo que tiene profundas implicaciones en el sentido de la formación, los aprendizajes y la forma de producir conocimiento, que en cada contexto tiene orígenes, debates e implicaciones diferentes.

\section{Referencias}

Arendt, Hannah. (2001). ¿Qué es la política? Barcelona: Ediciones Paidós Ibérica /Instituto de Ciencias de Educación de la Universidad Autónoma de Barcelona,

Bartolomé, Miguel. (2003). Las palabras de los otros: La antropología escrita por indígenas en Oaxaca. Cuadernos del Sur, Revista de Ciencias Sociales, 9 (18), Marzo 2003, pp. 24-50.

Coronado, Marcela. (2016). La cultura etnomagisterial en Oaxaca. Oaxaca: upN 201 Oaxaca.

Czarny, Gabriela. (Coord.) (2012). Jóvenes indígenas en la UPN. Relatos escolares desde la educación superior. México: UPN.

Dietz, Gunther y Mateos, Laura. (2010). La etnografía reflexiva en el acompañamiento de procesos de interculturalidad educativa: un ejemplo veracruzano. Revista Cuicuilco, núm. 48, enero-junio, pp. 107-131.

Honoré, Bernard. (1992). Vers l'ouvre de formation. L'ouverture à l'existence. Paris: L'Harmattan.

Magnan, Monique. (1997). Accompagner les parcours de formation. Lyon: CAFOC de Lyon.

Mateos, Laura, Mendoza, Rosa, y Dietz, Gunther. (2013). Diversidad e Interculturalidad en la educación superior convencional. En, M. Bertely, G. Dietz y G. Díaz Tepepa (coords.), Multiculturalismo y Educación. Estado del Conocimiento. México: SEP-ANUIES.

Mato, Daniel. (2017). Del "diálogo de saberes" a la construcción de modalidades de "colaboración intercultural": Aprendizajes y articulaciones más allá de la Academia. Lasa/Oxfam América 2017, Martin Diskin Memorial Lecture. Lasaforum, summer 2017: volume XLVIII: ISSUE 3.

Mato, Daniel. (2016). Educación Superior y Pueblos Indígenas y Afrodescendientes en América Latina. Interpelaciones, avances, problemas, conflictos y desafíos. En D. Mato (coord.). Educación Superior y Pueblos Indígenas en América Latina. Experiencias, interpelaciones y desafíos, (pp. 21-47). México: Universidad Nacional Autónoma de México.

Montaño, Ana (ed.) (2013). Educación Superior en América Latina: reflexiones y perspectivas en Educación. Bilbao: Universidad Deusto.

Navia, Cecilia. (2006). Autoformación de maestros en los márgenes del sistema educativo. Cultura, experiencia e interacción formativa. México: Pomares.

Navia, Cecilia y Hirsch, Ana. (2015). Ética profesional en estudiantes de posgrado en dos universidades mexicanas. Revista Electrónica de Investigación Edu- 
cativa, 17 (1). 100-115. Recuperado de http://redie.ens.uabc.mx/index.php/ redie/article/viewFile/495/1012

Pérez Ruiz, Maya. (coord.) (2011). Jóvenes indígenas y globalización en América Latina. Santiago de Chile: Fractal.

Prada, Fernando y López, Luis (2009). Educación superior y descentramiento epistemológico. En L. López. (coord.) Interculturalidad y educación superior. Perspcetivas latinoamericanas. Bolivia: FunProeib Andes-Plural.

Rea, Patricia. (2013). Educación superior, etnicidad y género. Zapotecas universitarios, profesionistas e intelectuales del Istmo de Tehuantepec en las ciudades de Oaxaca y México. Tesis Doctoral, ciesas, México, 2013.

Rebolledo, Nicanor. (coord.) (2014). La formación de profesionales de la educación indígena. Memorias, crónicas y voces de los formadores. México: upN.

Ricoeur, Paul. (2001). Del texto a la acción. Ensayos de hermenéutica II. México: FCE.

Rivera, Silvia. (2010). Ch'ixinakax utxiwa. Una reflexión sobre prácticas y discursos descolonizadores. Buenos Aires: Tinta Limón.

Salinas, Gisela. (2011). Formar docentes para la educación indígena: un acercamiento antropológico a una experiencia universitaria. Tesis de maestría. México: ENAH.

Santana, Yasmani. (2017). Los efectos de la diferencia étnica en programas de educación superior en México: educación intercultural en tensión. Revista nuestrAmérica, 5 (9), 59-76.

Suárez, Daniel. (2010). “Docentes, narrativa e investigación educativa. La documentación narrativa de las prácticas docentes y la indagación pedagógica del mundo y las experiencias escolares", en I. Sverdlick, (comp.), La investigación educativa. Una herramienta de conocimiento y de acción. Argentina: Noveduc.

Tubino, Fidel. (2007). Las ambivalencias de las acciones afirmativas. En J. Ansion, F. Tubino, S. Alfaro, M. González, L. Mujica, R. Segato y M. Villasante. Educar en ciudadanía intercultural. Experiencias y retos en la formación de estudiantes universitarios indígenas (pp. 91-110). Perú: Fondo Editorial de la Pontificia Universidad Católica del Perú.

Vasilachis, Irene. (2007) (Coord.) Estrategias de investigación cualitativa. Argentina: Gedisa.

Zapata, Claudia; Fernandes, Estevao y Del Valle, Emilio. (2017). Intelectuais indígenas nas Americas. Desafios y perspectivas. Revista de estudos e pesquisas sobre as Americas, 11 (2), pp. 1-4. 